\title{
Do Body Mass, Body Mass Index and Body Fat Ratio have an Effect on Proprioception?
}

\author{
Esra Ateş Numanoğlu ${ }^{1}$, Filiz Can ${ }^{1}$, Zafer Erden ${ }^{1}$ \\ ${ }^{1}$ Hacettepe University, Faculty of Health Sciences, Physiotheraphy and Rehabilitation Department, Ankara, TURKEY
}

Objectives: Proprioceptive sense plays important role of the protective reflex response against the harmful forces on the joint takes part in protection against the injuries. The load on the musculoskeletal system increases with higher body weights and joints become prone to injuries due to this increased load. The goal of this study was to investigate the relationship between the proprioceptive sense of the knee joint, mostly affected by loading, and the body weight, body mass index, and body fat ratio.

Methods: 25 healthy people aged between 21 and 36 years included in the study. Body weights, body mass indexes (BMI), and body fat ratios of the individuals were measured. Proprioceptive sense in the knee joint is evaluated through the active joint position sense (A-JPS) assessments. The A-JPS of 50 knees of 25 individuals are evaluated at supine and load bearing squat with $30^{\circ}$ and $90^{\circ}$ of knee flexion. To measure A-JPS, targeted angle were shown using goniometer and digital photography has been taken. Then they return to the initial position and repeat the angle and the second image was captured. After three times repetitions, deviations from $30^{\circ}$ and $90^{\circ}$ were called mean angle errors. The angle error measurements were performed with specifically written programme MATLAB (Computer Aided Design software). Pearson and Spearman Correlation Analyses were used for statistics. Results: There was no correlation between the body weight and the proprioceptive error at supine and squat for $30^{\circ}$ and $90^{\circ}$ flexion angles $(p>0.05)$. When the individuals has been grouped according to their BMI values thinnesses for $\left(B M I=15-20 \mathrm{~kg} / \mathrm{m}^{2}\right)$, normals for $\left(B M I=20-25 \mathrm{~kg} / \mathrm{m}^{2}\right)$, and overweights for $\left(B M I=25-30 \mathrm{~kg} / \mathrm{m}^{2}\right)$ and the correlation between the $\mathrm{BMI}$ and proprioceptive error has been evaluated, there was no correlation for thinnesses and normals, for overweights at $90^{\circ}$ squat position a medium strength positive correlation is observed $(r=0.644, p=0.01)$. For thinnesses and normals there is no correlation between the body fat ratio and the proprioceptive error. For the overweights for $30^{\circ}$ and $90^{\circ}$ flexions at squat there is medium strength, strong positive correlations ( $r 1=0.540, p=0.04 ; r 2=0.709, p=0.005)$.

Conclusion: Consequently, especially for individuals with high $\mathrm{BMI}$, at load bearing squat position for $90^{\circ}$ flexion, the proprioceptive sense decreases. Increased body fat ratio negatively affects the proprioception at load bearing squat position for $30^{\circ}$ and $90^{\circ}$ flexion angles. According to these results being overweight can be considered as an disadvantage in terms of proprioceptive sense.

The Orthopaedic Journal of Sports Medicine, 2(11)(suppl 3)

DOI: $10.1177 / 2325967114$ S00151

(C)The Author(s) 2014 